\title{
Arthroskopisch assistierte Versorgung dorsal dislozierter distaler intraartikulärer Speichenfrakturen - Technik und Ergebnisse
}

\section{Arthroscopically Assisted Osteosynthesis of Dorsally Tilted Intraarticular Distal Radius Fractures - Technique and Results}

\author{
Autoren \\ M. Lutz ${ }^{1}$, T. Wieland ${ }^{1}$, C. Deml ${ }^{2}$, S. Erhart ${ }^{2}$, A. Rudisch ${ }^{3}$, T. Klestil \\ Institute \\ ${ }^{1}$ Unfallchirurgie, Landesklinikum Baden/Mödling, Mödling, Österreich \\ ${ }^{2}$ Unfallchirurgie, Univ. Klinik Innsbruck, Innsbruck, Österreich \\ ${ }^{3}$ Radiologie, Univ. Klinik Innsbruck, Innsbruck, Österreich
}

\section{Schlüsselwörter \\ - Arthroskopie \\ - Radiusfraktur \\ - Gelenkstufe \\ - Bandverletzung \\ Key words \\ - arthroscopy \\ - radius fracture \\ - articular step off \\ - ligament injury}

eingereicht 19.6 .2014

akzeptiert 12.7.2014

\section{Bibliografie}

DOI http://dx.doi.org/

10.1055/s-0034-1385926

Handchir Mikrochir Plast Chir

2014; 46: 271-277

(c) Georg Thieme Verlag KG

Stuttgart · New York

ISSN 0722-1819

Korrespondenzadresse

Univ. Prof.

Dr. med. Martin Lutz

Unfallchirurgie

Landesklinikum Baden/Mödling

Sr. M.Restituta-Gasse 12

Mödling

Österreich 2340

martin.lutz@i-med.ac.at

\section{Zusammenfassung}

$\nabla$

Die vorliegende Arbeit beschreibt die Indikation und Technik der arthroskopisch unterstützten Verplattung dorsal dislozierter intraartikulärer Radiusfrakturen. Dabei wird auf ein bewährtes Repositionsverfahren eingegangen, die Klassifikation von Begleitverletzungen beschrieben und deren Therapiemöglichkeiten diskutiert. Klinische und radiologische Ergebnisse einer Fallserie von 17 Patienten werden präsentiert. Die DASHbzw. PRWE Werte lagen 2,5 Jahre nach der Operation bei 4,9 und 6,0. Die aktive Beweglichkeit in der Sagittalebene erreichte $123^{\circ}$ und somit $87 \%$ der gesunden Gegenseite, in der Frontalebene wurden $51^{\circ}$ erzielt, was $98 \%$ der Gegenseite entspricht. Die Unterarmdrehung war mit $163^{\circ}$ und $97 \%$ annähernd seitengleich. Der dorsopalmare Gelenkwinkel zum Ausheilungszeitpunkt betrug durchschnittlich $6^{\circ}$, der radioulnäre Gelenkwinkel $23^{\circ}$ und die ulnare Varianz -1,2 mm. Der skapholunäre Gelenkspalt wurde mit $1,6 \mathrm{~mm}$, der skapholunäre Gelenkwinkel mit $57^{\circ}$ gemessen.

\section{Einleitung}

\section{$\nabla$}

Für junge aktive Patienten gilt die anatomische Wiederherstellung des distalen Radius nach Speichenfrakturen als Voraussetzung für eine funktionelle Wiederherstellung. Nur dadurch wird eine physiologische Kraftübertragung im Handgelenk mit entsprechender Druckverteilung zwischen Radius und Ellenkopf sowie ein ungestörtes karpales Alignment erzielt [1-5].

Während für Gelenkfrakturen anderer anatomischer Regionen die offene Reposition unter Sicht mit exakter Gelenkflächenrekonstruktion gefordert wird, haben sich am Handgelenk weitgehend indirekte, bildwandlergesteuerte Repositionstechniken etabliert. Dabei wird auf eine direkte Einsicht auf die Gelenkfläche verzichtet [6,7].

\section{Abstract \\ $\nabla$}

The present paper describes the indication and application of an arthroscopically assisted osteosynthesis for distal radius fractures. Visualisation of articular incongruency is emphasised with special regard to articular fracture fragment reduction. In addition to that, classification of soft tissue injuries and treatment options are discussed. The final clinical and radiological results of 17 patients are presented: DASH and PRWE averaged 4.9 and 6.0 respectively. Active range of motion measured $123^{\circ}$ for flexion/extension, $51^{\circ}$ for radial and ulnar deviation and $163^{\circ}$ for pronosupination, which is $87 \%, 98 \%$ and $97 \%$, respectively, compared with the opposite wrist. Radial inclination at final follow-up was $23^{\circ}$, palmar tilt measured $6^{\circ}$ and ulnar variance averaged $-1.2 \mathrm{~mm}$. The scapholunate gap at follow-up was $1.6 \mathrm{~mm}$, and the scapholunate angle measured $57^{\circ}$.

Eine Besonderheit stellt am Radiokarpalgelenk der sehr komplex angeordnete Bandapparat dar, dessen Verletzung in einer schwerwiegenden Gefügestörung des Handgelenkes enden kann.

Somit bleibt nur jener dorsoradiale Gelenkanteil, an dem die Gelenkkapsel am Radius ansetzt, als potentielles Fenster für eine Arthrotomie. Erfahrungsgemäß bietet dieses Fenster aber nur eine ungenügende Einsicht in das Gelenk.

Ausgehend von diesen Überlegungen bietet die $\mathrm{Ar}$ throskopie die Möglichkeit die gesamte radiokarpale Gelenkfläche zu inspizieren, ohne dabei gelenktragende Fragmente zu denudieren bzw. entscheidende Bandstrukturen zu schädigen [8,9]. Begleitverletzungen, wie sie sowohl in experimentellen als auch klinischen Studien in einem hohen Prozentsatz nachgewiesen werden konn- 
ten, werden gleichzeitig erfasst und bei Bedarf mitbehandelt [10].

\section{Indikation und Technik \\ $\nabla$}

Die arthroskopisch assistierte Versorgung der distalen intraartikulären Speichenfraktur ist bei aktiven Patienten bis zum 65 . Lebensjahr und bei Frakturformen mit 2 bzw. 3 gelenktragenden Fragmenten kombiniert mit einer dorsalen Trümmerzone indiziert. Dies entspricht C1-, C2- und einfachen C3-Frakturen nach der AO-Klassifikation.
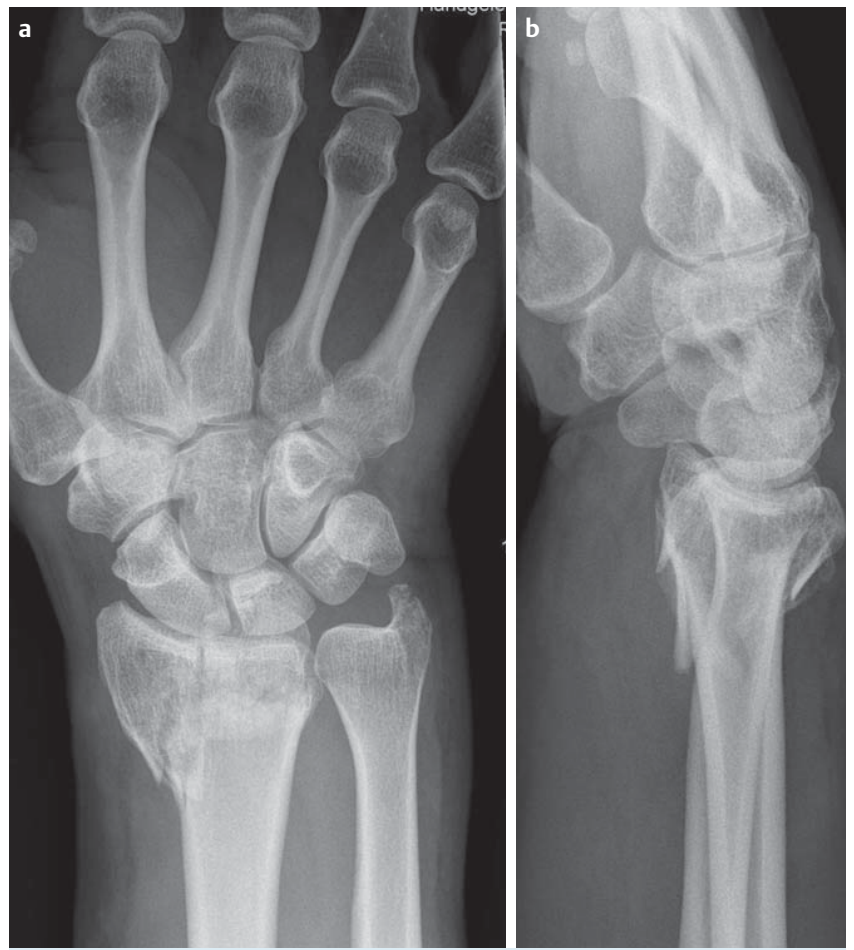

Abb. 1 a, b Unfallröntgenbild: dorsal dislozierter intraartikulärer Speichenbruch.
Die operative Versorgung erfolgt zwischen dem 2. und 6. Tag nach Trauma, initial wird in allen Fällen eine gedeckte Reposition in Bruchspaltanästhesie durchgeführt und eine dorsale Gipsschiene angelegt. Die präoperative Abklärung wird durch eine Feinschicht-Computertomografie vervollständigt ( $\bullet$ Abb. 1, 2). Das palmare ulnare Kantenfragment, welches offen reponiert wird, gilt dabei als Schlüsselfragment, das die Länge des Radius und die Kongruenz im distalen Radioulnargelenk vorgibt. An diesem Fragment orientiert sich die weitere Gelenkrekonstruktion. Die Retention der Fraktur erfolgt über eine von palmar eingebrachte winkelstabile Platte.

Der (die) Patient(-in) wird am Rücken gelagert und die betroffene Extremität am Handtisch ausgelagert. Für den arthroskopischen Teil der Operation wird eine Extensionsvorrichtung an der frakturabgewandten Seite des Operationstisches montiert, über die das Handgelenk vertikal und in Mittelstellung zwischen Pround Supination extendiert wird. Die Assistenz übt während dieses Operationsschrittes einen kontinuierlichen Gegenzug am Oberarm aus.

Der Arthroskopieturm wird auf der Gegenseite des verletzten Handgelenkes in Höhe des Beckens positioniert, der Bildwandler auf die Seite des verletzten Handgelenkes gestellt.

Im ersten Operationsschritt wird ein palmarer Zugang über der Sehne des Musculus flexor carpi radialis angelegt, die weitere Präparation erfolgt in konventioneller Weise bis an den Knochen mit Freilegung der metaphysären Querfraktur und dem fakultativen Ausläufer in das Radiokarpalgelenk [6].

Nun wird die Reposition des Gelenkblockes durchgeführt, wobei auf ein exaktes metaphysäres Alignment geachtet und bei Vorliegen eines palmar ulnaren Kantenfragmentes dies isoliert temporär fixiert wird. Diese Stabilisierung erfolgt mit $1,4 \mathrm{~mm}$ Kirschner-Drähten, die von distal her über die Gelenklippe in die Metaphyse vorgebohrt und dort verankert werden. Ein zusätzlicher Bohrdraht stabilisiert den Gelenkblock über den Processus styloides radii. Eine winkelstabile Radiusplatte wird beugeseitig angelegt und zu diesem Zeitpunkt der Operation lediglich proximal der Fraktur mit Schrauben fixiert ( $\bullet$ Abb. 3).

Nach Kontrolle dieser ersten Reposition im Bildwandler wird das Handgelenk unter Zuhilfenahme von 2 Mädchenfängern vertikal
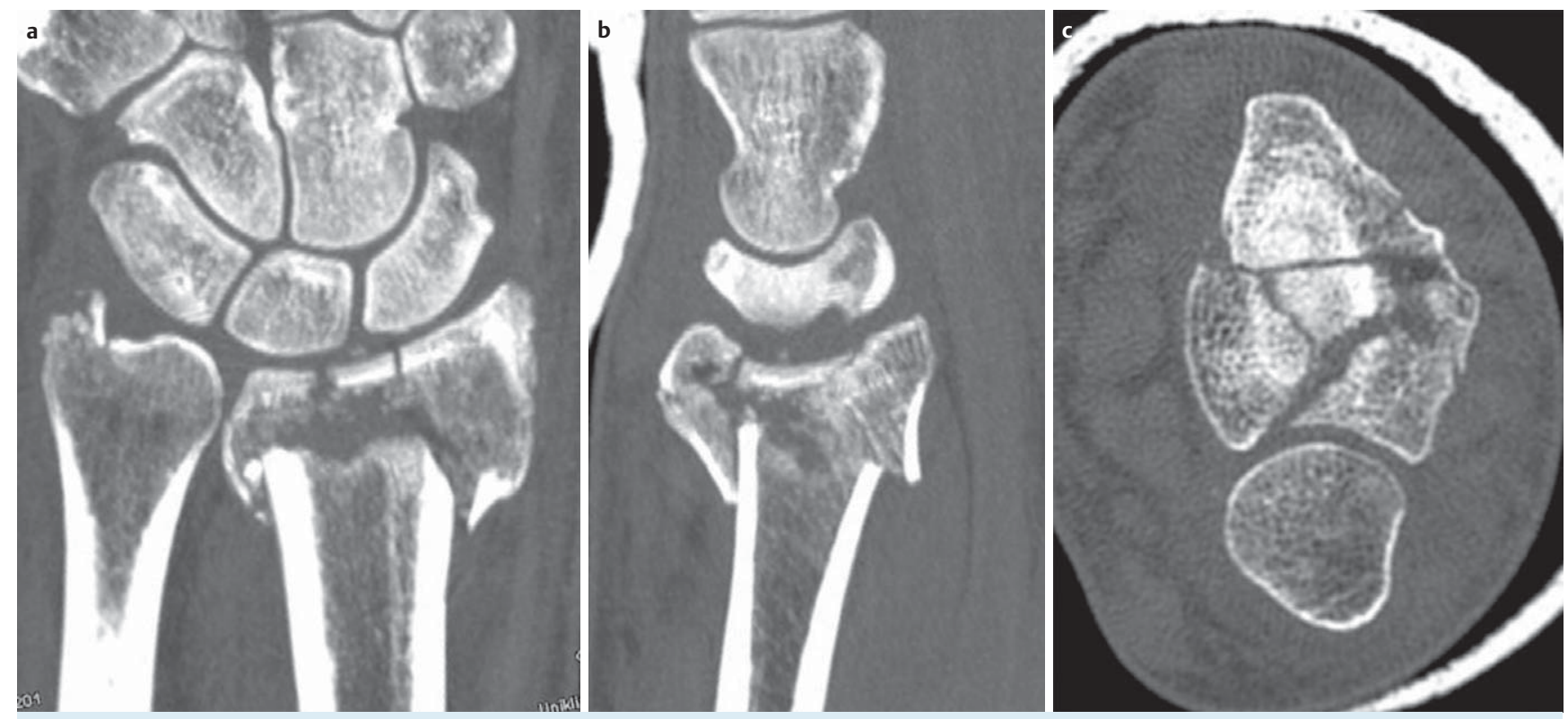

Abb. 2 a, b, c Computertomografie nach Reposition; coronar, seitlich, axial. 


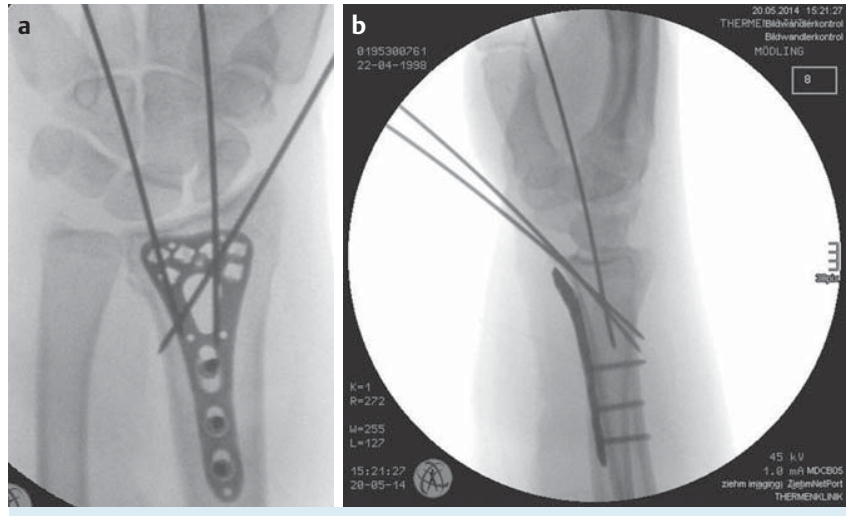

Abb. 3 a, b Intraoperative Bildwandlerkontrolle nach temporärer Kirschner-Drahtfixation der Fraktur von beugeseitig und radial. mit $5 \mathrm{~kg}$ Extensionsgewicht in den Aushang gebracht. Durch die vertikale Orientierung des Unterarmes stellt sich das DRU Gelenk in Mittelstellung zwischen Pro- und Supination ein, was Vorteile für die Reposition des dorsoulnaren Kantenfragmentes mit sich bringt, da die Spannungsverhältnisse des ulnokarpalen Komplexes (TFCC) geringer als in Pronation sind und der Ellenkopf zentral mit der Incisura ulnaris des Radius artikuliert.

Die Standardportale für die arthroskopische Versorgung sind das 3/4-Portal initial für das Arthroskop, das 6R-Portal wird im ersten Schritt als Instrumentenzugang geführt. Die Autoren arthroskopieren mit einer $2,7 \mathrm{~mm} 30^{\circ}$ Optik im flüssigen Milieu mit einem Pumpendruck von 30 bis maximal $40 \mathrm{~mm} \mathrm{Hg}$, wobei auf einen suffizienten Abfluss über das zweite Portal, ansonsten über eine Abflusskanüle, geachtet wird. Alternativ dazu kann auch die Trocken-Arthroskopie zur Anwendung kommen. Das Risiko eines Kompartment-Syndroms ist bedingt durch den beugeseitigen Zugang für die Plattenosteosynthese als gering einzuschätzen und nie beobachtet worden [8].

Mit dem 3mm Shaver werden Koagel, Kapselanteile sowie etwaige Knorpel-Knochenfragmente aus dem Gelenk entfernt. Im Bereich der Fossa scaphoidea werden Knorpelüberzug von Skaphoid und Radius sowie die Bandursprünge des Radio-skapho-Kapitatum-Bandes und langen radiolunären Bandes beurteilt. Nach ulnar hin wird der skapholunäre Bandapparat und das radioskapho-lunäre Band ausgetastet. Im Anschluss werden die Fossa lunata mit der verbliebenen Fehlstellung des dorsoulnaren Kantenfragmentes, bzw. die Fraktur zwischen Fossa scaphoidea und Fossa lunata dokumentiert. Ulnar wird der begleitende Schaden des TFCC erhoben, der lunotriquetrale Bandapparat ausgetastet und das Os triquetrum inspiziert. Arthroskop- und Instrumentenzugang können dabei wahlweise getauscht werden [11]. Die typische intraartikuläre Fehlstellung stellt eine Verkippung der Gelenkfragmente zwischen Fossa scaphoidea und Fossa lunata dar, bzw. eine Gelenkstufe zwischen palmarem und dorsoulnarem Kantenfragment ( $\bullet$ Abb. 4).

Neben diesen Gelenkstufen sollte auch die Kurvatur der Gelenkfläche beachtet werden, um ein zentrales Einsinken in Form einer ,Gelenkwanne‘ zu vermeiden. Dabei orientiert sich das dorsoulnare Kantenfragment am palmaren Kantenfragment. Eine obligate Wiederherstellung des dorsopalmaren Gelenkwinkels von plus $10^{\circ}$ wird nicht angestrebt, $0^{\circ}$ sind funktionell durchaus akzeptabel. Durch die korrekte Kurvatur der distalen Speichengelenkfläche in der Sagittalebene wird der Dreh-Gleitmechanismus des Radiokarpalgelenkes insbesonders die Beweglichkeit

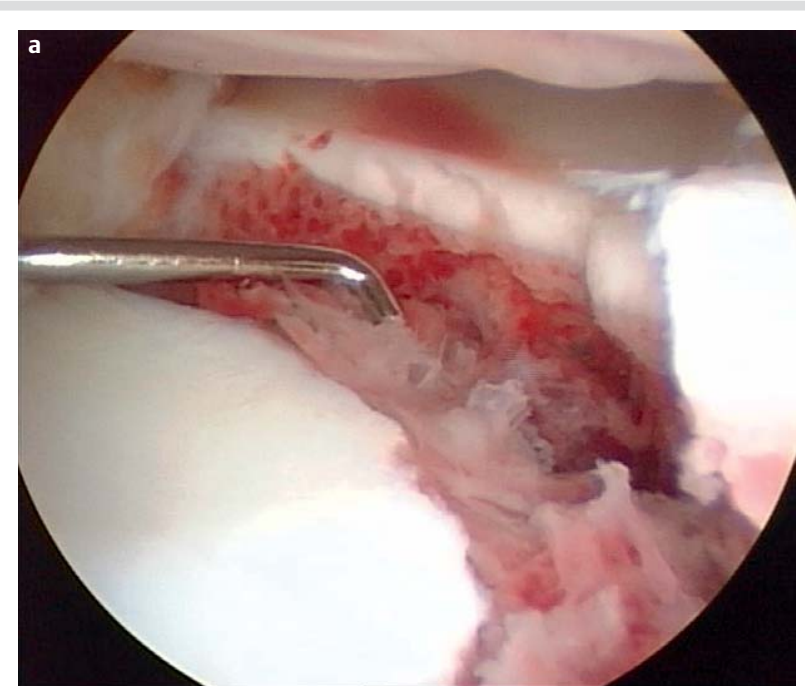

b

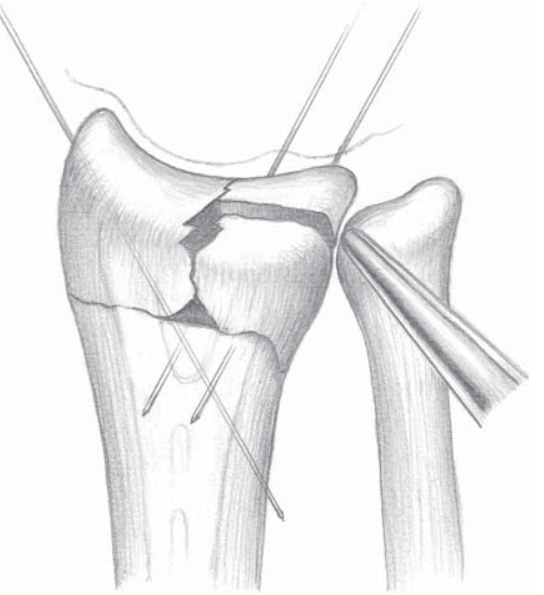

Abb. 4 a, b Intraoperatives arthroskopisches Bild der Gelenkstufe. Schematisierte Darstellung der Gelenkstufe von streckseitig.

zwischen Os lunatum und Fossa lunata des Radius gewährleistet $[12,13]$.

Bei Vorliegen einer Gelenkstufe wird nun von ulnar her ein doppelt gespitzter Kirschner-Draht subchondral in das radiale Hauptfragment gebohrt, radial perkutan ausgeleitet und bis zur artikulären Gelenkstufe zurückgezogen. Im Anschluss werden Kirschner-Drähte derselben Stärke in die dorsalen noch mobilen Gelenkfragmente eingebracht und als Joy sticks zur Feinreposition genutzt. Ist die Reposition der Gelenkfragmente abgeschlossen wird der erste Kirschner-Draht von radial her in das dorsoulnare Fragment in Rückbohrtechnik gelegt und im Anschluss ein weiterer Bohrdraht von dorsal in das palmare Kantenfragment gelegt ( $\bullet$ Abb. 5, 6).

Damit gelingt in der Regel eine suffiziente temporäre Fixation der Gelenkfragmente, wobei auch Frakturen, die in die Incisura ulnaris des Radius einlaufen anatomisch reponiert sind.

Nun werden die Begleitverletzungen des TFCC sowie der karpalen Bänder angegangen. Zentrale Risse des TFCC werden debridiert, radialseitige Abrisse fakultativ transossär refixiert und ulnarseitige Abrisse belassen [14]. Eine midkarpale Arthroskopie zur Klassifikation eines skapholunären bzw. lunotriquetralen Bandschadens entsprechend der Geissler-Klassifikation schließt den arthroskopischen Teil des Verfahrens ab [15]. Eine Grad-4Läsion, somit eine Zerreißung des gesamten skapholunären 


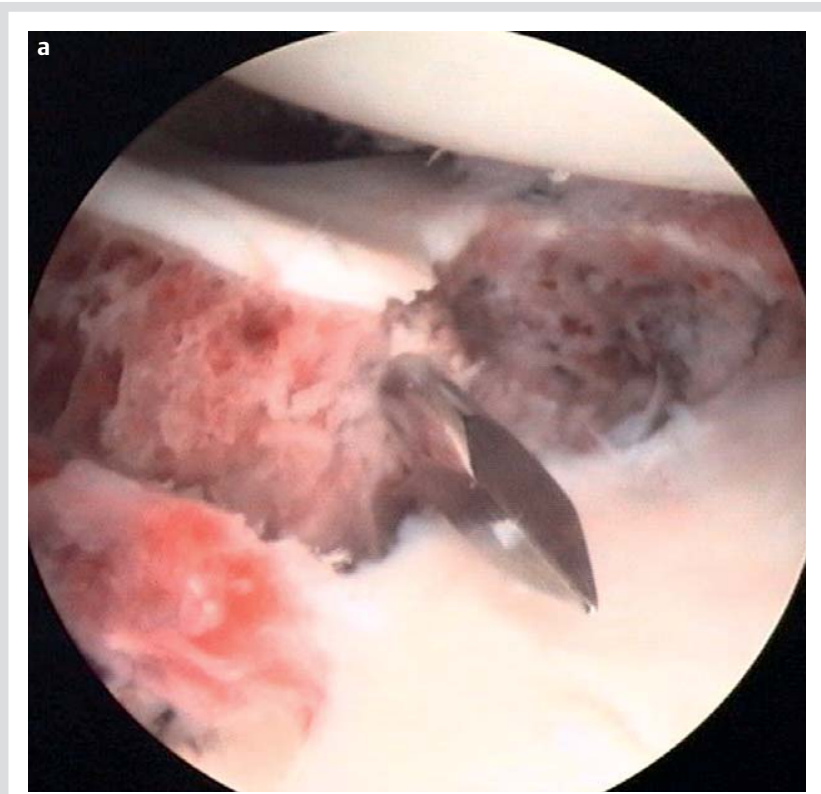

b

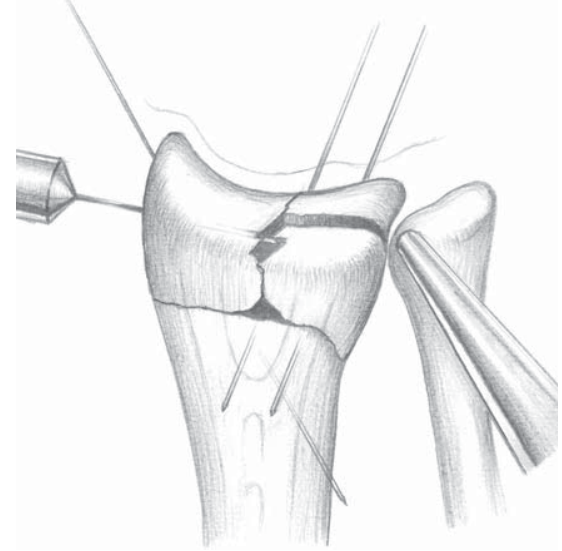

c

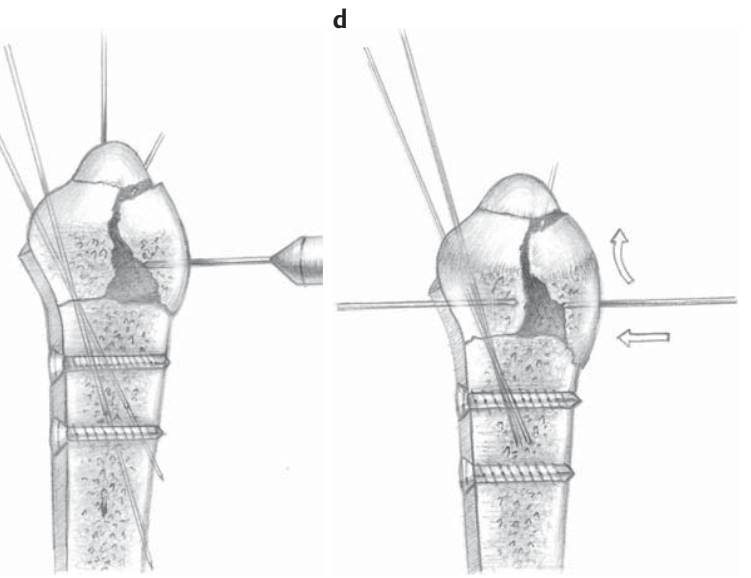

Abb. 5 a, b, c, d Intraoperatives arthroskopisches Bild der Gelenkstufe mit vorgelegtem Kirschner-Draht. Schematisierte Darstellung der Repositionstechnik von streckseitig und seitlich.

Bandes wird dabei im Falle intakter Knorpelverhältnisse in gleicher Sitzung mittels offener transossärer Reinsertion versorgt sowie die Handwurzel stifttransfixiert. Dabei wird vor allem auf die Refixation des dorsalen, biomechanisch stärksten Anteiles des SL-Bandes Wert gelegt ( $\bullet$ Tab. 1, 2).

Für die Grad-3-Läsion kommen die temporäre perkutane Kirschner-Drahtfixation oder die konservative Therapie in Form der Gipsruhigstellung in Frage $[10,16]$. Im hier vorgestellten Kollek-

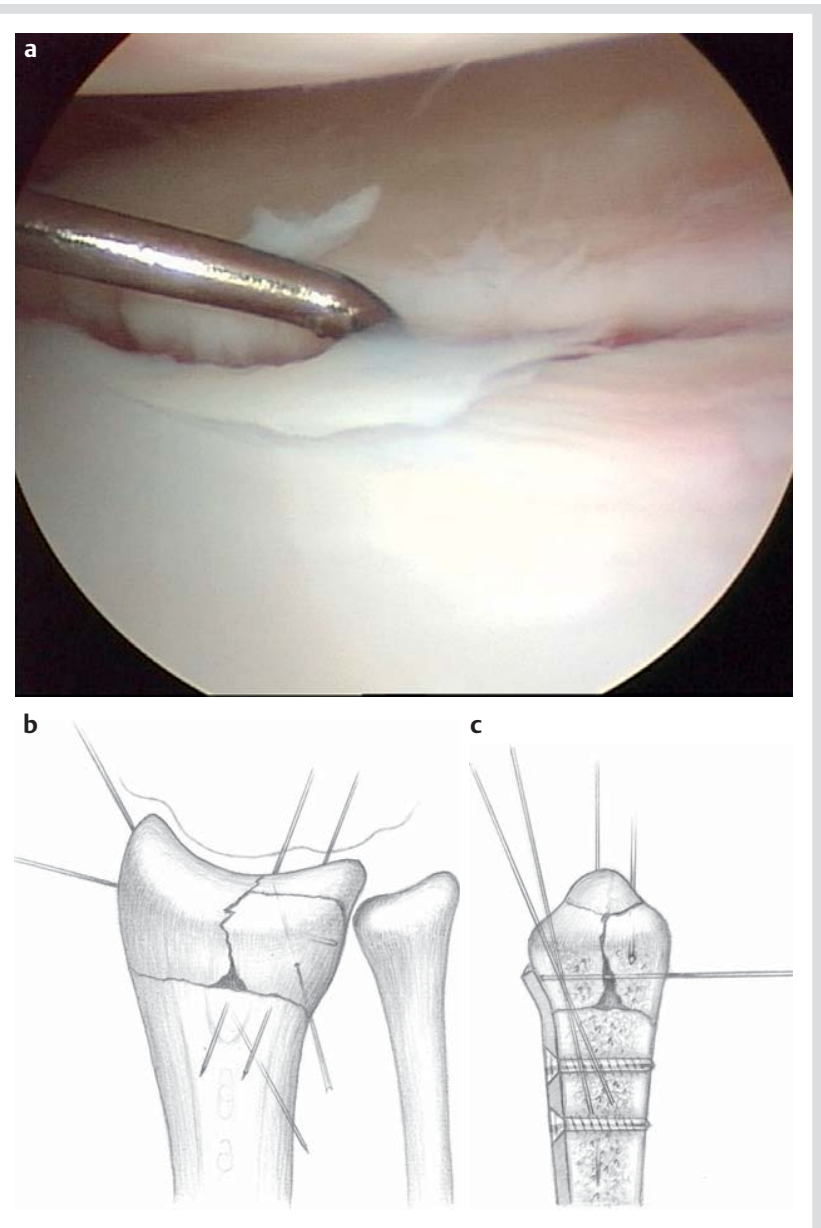

Abb. 6 a, b, c Intraoperatives arthroskopisches Bild nach Reposition der Gelenkfragmente. Schematisierte Darstellung der Reposition und Kirschner-Drahtfixation von streckseitig und seitlich.

Tab. 1 Stadieneinteilung der interkarpalen Bandschäden nach Geissler.

$\begin{array}{ll}\text { Verletzungsgrad } & \text { Beschreibung } \\ \text { Grad 1 } & \text { Bandapparat blutig imbibiert } \\ \text { Grad 2 } & \begin{array}{l}\text { Mit demTasthäkchen kann zwischen die Karpalkno- } \\ \text { chen eingegangen werden }\end{array} \\ \text { Grad 3 } & \begin{array}{l}\text { Mit dem Tasthäkchen kann zwischen die Karpalkno- } \\ \text { chen eingegangen werden und das Häkchen rotiert } \\ \text { werden }\end{array} \\ \text { Grad 4 } & \begin{array}{l}\text { Die Optik kann zwischen die Karpalknochen von } \\ \text { midkarpal nach radiokarpal vorgeschoben werden }\end{array}\end{array}$

tiv wurden Grad-3-Rupturen konservativ im Gipsverband für 4 Wochen ruhiggestellt.

Im Anschluss werden die distalen Plattenschrauben besetzt und die temporär eingebrachten Kirschner-Drähte im Bereich des distalen Radius sukzessive entfernt. Eine abschließende Bildwandlerkontrolle dokumentiert das Ergebnis im anteroposterioren, seitlichen und axialen Strahlengang [17]. Eine fraglich intraartikuläre Schraubenlage kann nun noch arthroskopisch kontrolliert werden ( $\bullet$ Abb. 7).

\section{Patienten}

17 von insgesamt 23 Patienten, 13 Frauen und 4 Männer mit einem Durchschnittsalter von 49 Jahren, die im Zeitraum 
Tab. 2 Palmer Klassifikation der akuten TFCC Verletzungen.

\begin{tabular}{|ll} 
Verletzungsgrad & Beschreibung \\
\hline 1A & Zentraler Riss des Diskus \\
\hline 1B & Abriss des TFCC von der Ulna \\
1C & Abriss der ulnokarpalen Ligamente \\
\hline 1D & Abriss des TFCC vom Radius \\
\hline
\end{tabular}
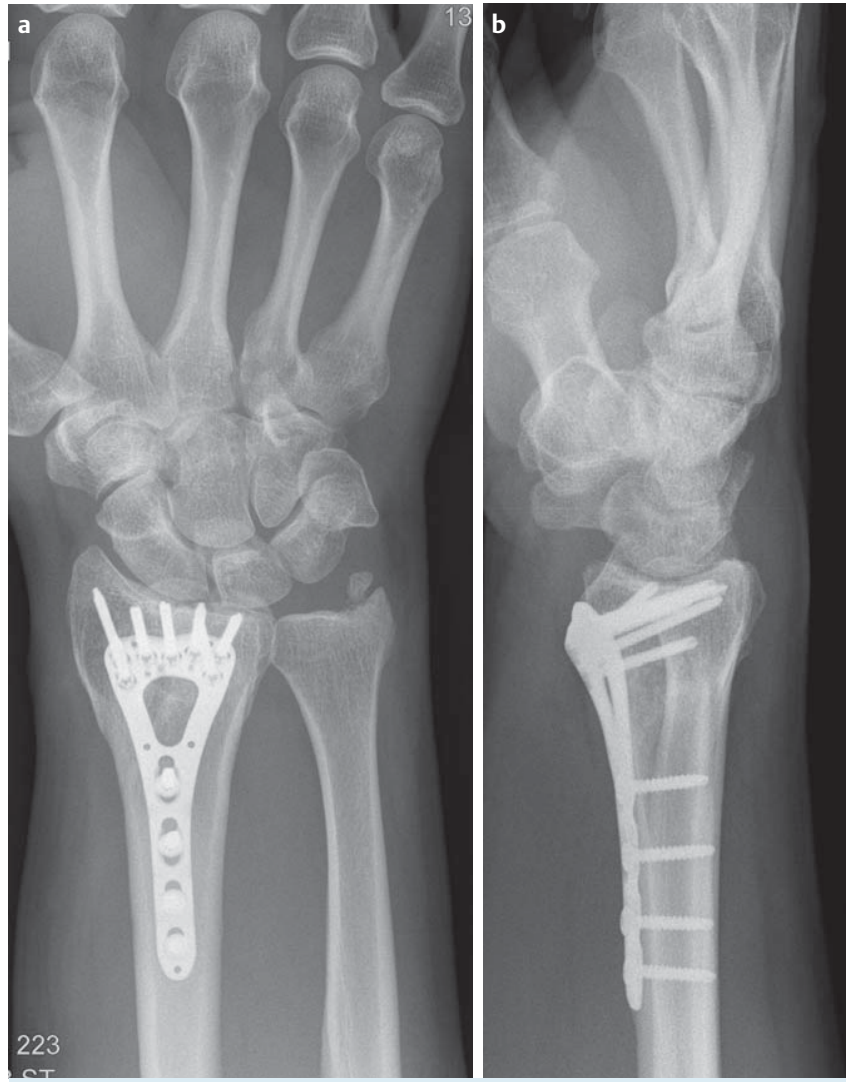

Abb. 7 a, b Röntgenologisches Ausheilungsbild der Fraktur in 2 Ebenen.

2011/2012 in der genannten Technik operativ versorgt wurden, konnten klinisch nach durchschnittlich 31 Monaten nachkontrolliert werden.

Folgende Frakturverteilung lag im vorgestellten Kollektiv vor:

C1-Frakturen 3 Patienten

C2-Frakturen 7 Patienten

C3-Frakturen 7 Patienten

Die arthroskopischen intraoperativen Befunde wurden einerseits hinsichtlich der Gelenkstufen ausgewertet, andererseits wurden die ligamentären karpalen Begleitverletzungen entsprechend der Geissler-Klassifikation erhoben und Diskuspathologien nach Palmer klassifiziert.

Zum Nachuntersuchungszeitpunkt wurde die aktive Beweglichkeit in der Frontal- und Sagittalebene sowie die Unterarmumwendebewegung im Seitenvergleich gemessen. Zusätzlich erfolgte eine Messung der Grobkraft ebenfalls im Seitenvergleich sowie die Ermittlung des Schmerzes über die VAS-Skala.

Der DASH-Score und der Patient Rated Wrist Evaluation Score vervollständigen die klinische Bewertung.

Die Röntgenverlaufsbilder wurden hinsichtlich der erzielten artikulären Kongruenz vermessen. Zudem wurde der dorsopalmare und radioulnäre Gelenkwinkel im Ausheilungsbild ermittelt und eine Pseudarthrose des Ellengriffels dokumentiert. Die
Messung des skapholunären Gelenkspaltes und des skapholunären Winkels ergänzten die Auswertung.

\section{Ergebnisse}

Durch die Arthroskopie konnte in allen Fällen einer verbliebenen Inkongruenz der Gelenkfläche eine Verbesserung erzielt werden. Im Detail lagen folgende Ergebnisse vor und nach dem arthroskopischen Operationsschritt vor:

Arthroskopische Gelenkstufe nach bildwandlergestützter Reposition:

0-1mm 7 Patienten

1-2mm 9 Patienten

über $2 \mathrm{~mm} 1$ Patient

Arthroskopische Gelenkstufe nach arthroskopischer Reposition:

0-1 mm 17 Patienten

Die ligamentären interkarpalen Verletzungen und Verletzungen des TFCC waren wie folgt verteilt:

SL-Bandrupturen nach Geissler

Grad 28 Patienten

Grad 36 Patienten

Grad 41 Patient

LT-Bandruptur nach Geissler

Grad 22 Patienten

TFCC-Ruptur nach Palmer

$1 \mathrm{~A} 3$ Patienten

1B 5 Patienten

1D 3 Patienten

Prozessus styloides ulnae Frakturen lagen bei 12 Patienten vor, wobei eine Kombination von TFCC-Ruptur und Fraktur in 7 Fällen beobachtet wurde.

Die aktive Beweglichkeit im Handgelenk zum Nachuntersuchungszeitpunkt betrug für die Extension $68^{\circ}(60-80)$, für die Flexion $55^{\circ}$ (45-70), die Radialduktion wurde mit $22^{\circ}(15-30)$ die Ulnarduktion mit $29^{\circ}(20-40)$ gemessen. $81^{\circ}(80-85)$ wurden für die Supination und $83^{\circ}$ (70-90) für die Pronation ermittelt. Auf der unverletzten Gegenseite wurden folgende Werte erhoben:

Extension $74^{\circ}(60-90)$

Flexion $67^{\circ}(55-80)$

Radialduktion $21^{\circ}(15-30)$

Ulnarduktion $31^{\circ}(25-40)$

Supination $82^{\circ}(80-90)$

Pronation $85^{\circ}(80-90)$

Somit wurden für alle Teilbewegungen bis auf die Flexion mehr als $90 \%$ des Bewegungsumfanges der unverletzten Gegenseite erzielt. Die Flexionseinschränkung betrug im Mittel 17\%.

Die Grobkraft beim Faustschluss wurde durchschnittlich mit 28 $\mathrm{kg}(16-48 \mathrm{~kg})$ gegenüber $29 \mathrm{~kg}(18-50 \mathrm{~kg})$ auf der unverletzten Gegenseite gemessen und erzielte damit $96 \%$.

Auf der 10-teiligen VAS-Skala gaben 3 Patienten in Ruhe einen Schmerzgrad 1 an, während 14 Patienten schmerzfrei waren.

Bei Belastung waren 8 Patienten schmerzfrei, 4 bewerteten ihre Schmerzen mit 1, zwei Patienten mit 2 und 3 Patienten mit 3.

Für den DASH konnte in diesem Kollektiv ein Wert von 4,9 $(0-18,3)$ und für den PRWE ein Wert von 6,0 (0-20,5) erhoben werden.

An den Ausheilungsbildern wurde der radioulnare Gelenkwinkel mit durchschnittlich $23^{\circ}$ (17-28) und der dorsopalmare Gelenkwinkel mit $6^{\circ}(1-12)$ gemessen. Die ulnare Varianz betrug im Mittel -1,2 mm (-4,1- +2,5), der skapholunäre Gelenkspalt 
im anteroposterioren Strahlengang $1,6 \mathrm{~mm}(0,8-2,5)$ und der skapholunäre Gelenkwinkel im seitlichen Bild 57²(36-79). Eine Pseudarthrose des Ellengriffels lag in 8 Fällen vor.

\section{Diskussion}

Die Therapie der Wahl für die Behandlung des instabilen, distalen intraartikulären Speichenbruches ist die anatomische Wiederherstellung von Länge und Form, sowie die exakte Rekonstruktion der Gelenkfläche [3]. In klinischen wie auch experimentellen Studien konnte gezeigt werden, dass verbliebene Inkongruenzen in Form von Gelenkstufen bzw. Gelenkverbreiterung zu einer Zunahme der Stresskonzentration, Bewegungsstörungen und langfristig radiokarpaler Arthrose führen [1,5,17].

Neben diesen Veränderungen ist die Kurvatur der distalen Speichengelenkfläche in der Sagittalebene speziell auf Höhe der Fossa lunata entscheidend. Ein zentrales Einsinken in Form einer Gelenkwanne resultiert hier in einer Störung des Dreh-Gleitmechanismus des Os lunatum auf der Radiuskonsole. Dies hat zur Folge, dass einerseits die Sagittalbewegung der proximalen Handwurzelreihe eingeschränkt ist, andererseits die Druckübertragung zwischen Lunatum und Radius vom ursprünglich palmaren Gelenkdrittel auf die dorsale Gelenklippe ausgeweitet wird $[12,13]$.

In der klinischen Anwendung der Handgelenksarthroskopie bei intraartikulären Speichenbrüchen hat sich gezeigt, dass das intraoperative Bildwandlerbild in vielen Fällen nicht in der Lage ist, entsprechende Gelenkstufen bzw. Lücken in der radiokarpalen Gelenkfläche darzustellen. Das Bildwandlerergebnis unterschätzt regelmäßig das wahre Ausmaß der Gelenkinkongruenz $[18,19]$. Dabei korrelieren diese Gelenkstufen bzw. Lücken mit einem schlechteren funktionellen Endergebnis [2].

Beim isoliert offenen Vorgehen gibt es zwar die Möglichkeit über eine dorsoradiale Arthrotomie Einsicht in das Gelenk zu erhalten, der Überblick ist allerdings häufig unbefriedigend und kann lediglich durch Einkerben des radiokarpalen Bandapparates verbessert werden [21]. Untersuchungen zur Auswirkung dieser Arthrotomie haben gezeigt, dass Arthrofibrosen mit diesem Vorgehen vergesellschaftet sind und zudem Durchblutungsstörungen der streckseitigen Gelenkfragmente ausgelöst werden können.

Im Gegensatz dazu ermöglicht die Arthroskopie einen ausgezeichneten Gelenküberblick unter Schonung der Blutversorgung der streckseitigen Gelenkfragmente. Eine exakte Reposition der Gelenkfläche kann durchgeführt werden und ligamentäre Begleitverletzungen können diagnostiziert und in gleicher Sitzung behandelt werden [9]. Im Falle multipler artikulärer Fragmente sind der Rekonstruktion zwar Grenzen gesetzt, doch lassen sich 2- bzw 3-Fragment-Frakturen durch die beschriebene Technik exakt einrichten, und auch bei artikulären Mehrfragmentfrakturen lässt sich meist eine Verbesserung in der Kongruenz der Gelenkfläche erzielen. Ein zusätzlicher Vorteil gegenüber der konventionellen Technik ist die anatomische Wiederherstellung des DRU-Gelenkes mit radialseitig entsprechender Ausspannung des TFCC.

Kontrovers wird in der Literatur derzeit die Behandlung der Begleitverletzungen diskutiert. Für komplette Rupturen interkarpaler Bänder gilt die Rekonstruktion als Therapie der Wahl. Ob für Teilrupturen aber die perkutane Kirschner-Drahttransfixation bessere Ergebnisse als die konservative Therapie mittels Ruhigstellung ergibt, werden zukünftige Studien zeigen müssen $[9,10]$.
Ähnliches gilt für die Mitbehandlung ulnarseitiger Pathologien. Während einige Autoren die Refixation radial- bzw. ulnarseitiger Ausrisse des TFCC empfehlen, wurden für begleitende Processus styloides ulnae Frakturen widersprüchliche funktionelle Ergebnisse ermittelt. Sammer konnte keinen Zusammenhang zwischen knöchernen ulnarseitigen Verletzungen und klinischem Endergebnis finden [22]. Im Gegensatz dazu stellten Krämer und Mitarbeiter fest, dass das klinische Ergebnis durch eine Prozessus styloides ulnae Fraktur negativ beeinflusst wird [23].

Im Behandlungsregime der Autoren genießt die stufenfreie Wiederherstellung der Gelenkfläche die höchste Priorität. Komplette interkarpale Bandrupturen werden im Zuge des Eingriffes rekonstruiert, Partialrupturen konservativ behandelt. Rupturen des TFCC werden bis auf jene Fälle, die für ein zentrales Debridement in Frage kommen, ebenfalls konservativ behandelt.

Obwohl das hier vorgestellte Kollektiv klein ist und eine entsprechende Vergleichsgruppe fehlt, geht doch hervor, dass die Ergebnisse überzeugend sind. Die arthroskopische Feinreposition macht möglich, was für Gelenkfrakturen anderer anatomischer Regionen gefordert wird, nämlich die stufenfreie wasserdichte Reposition der Gelenkfläche unter Sicht [24].

Ein weiter differenziertes Vorgehen im Bereich der Band- bzw. Diskuspathologien werden zukünftige Studien erbringen müssen.

\section{Danksagung}

Die Autoren danken der Ergotherapie Lechner in Innsbruck für die Erhebung der Funktions- und Kraftmessung der Patienten zum Nachuntersuchungszeitpunkt.

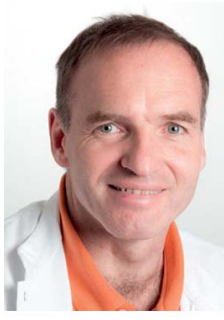

Martin Lutz geb. 22.08.1966 in Kufstein Studium an der medizinischen Fakultät der Universität Innsbruck von 1984-1991. Postpromotionelle Ausbildung am Institut für Anatomie Innsbruck (Univ. Prof. Platzer) 1991 und 1992. Ab Mai 1992 Turnusausbildung am Bezirkskrankenhaus in Wörgl (Tirol). Ab 1993 Facharztausbildung Unfallchirurgie an der Univ. Klinik für Unfallchirurgie Innsbruck (Univ. Prof. Beck) mit spezieller Berücksichtigung der Handchirurgie (Univ. Doz. Pechlaner). Ab 1998 Facharzt, ab 2003 Oberarzt und 2004 Habilitation zum Thema: Luxationen und Luxationsfrakturen kleiner Gelenke.

Auslandsaufenthalt am Princess Alexandra Hospital in Brisbane 2009/2010;

Stellvertretender Direktor an der Univ. Klinik für Unfallchirurgie Innsbruck 2011/2012. Von Juli 2012 bis Juni 2013 Praxisgemeinschaft Unfallchirurgie in Innsbruck. Ab Juli 2013 Oberarzt an der Unfallchirugie des Landesklinikums Baden/ Mödling (Univ. Prof. Klestil) (Niederösterreich).

\section{Interessenkonflikt: Nein}

\section{Literatur}

1 Baratz ME, Des Jardins J, Anderson DD et al. Displaced intra-articular fractures of the distal radius: the effect of fracture displacement on contact stresses in a cadaver model. J Hand Surg [Am] 1996; 21: 183-188

2 Gliatis JD, Plessas SJ, Davis TR. Outcome of distal radial fractures in young adults. J Hand Surg [Br] 2000; 25: 535-543 
3 McQueen MM, Caspers J. Colles fracture: does the anatomical result affect the final function? J Bone Joint Surg [Br] 1988; 70: 649-651

$4 \mathrm{Ng} \mathrm{CY} \mathrm{1,} \mathrm{McQueen} \mathrm{MM.} \mathrm{What} \mathrm{are} \mathrm{the} \mathrm{radiological} \mathrm{predictors} \mathrm{of} \mathrm{func-}$ tional outcome following fractures of the distal radius? J Bone Joint Surg [Br] 2011; 93: 145-150

5 Trumble TE, Schmitt SR, Vedder NB. Factors affecting functional outcome of displaced intra-articular distal radius fractures. J Hand Surg [Am] 1994; 19: 325-340

6 Pechlaner S. Distal intra-articular radius fractures. Indications for and technique of open reduction and plate osteosynthesis. Orthopäde 1993; 22: 46-51

7 Ruch DS, Vallee J, Poehling GG et al. Arthroscopic reduction versus fluoroscopic reduction in the management of intra-articular distal radius fractures. Arthroscopy 2004; 20: 225-230

8 del Pinal F. Dry arthroscopy and its applications. Hand Clin 2011; 27: 335-345

9 Geissler WB, Freeland AE. Arthroscopic management of intra-articular distal radius Fractures. Hand Clin 1999; 15: 455-465

10 Forward DP, Lindau TR, Melsom DS. Intercarpal ligament injuries associated with fractures of the distal part of the radius. J Bone Joint Surg [Am] 2007; 89: 2334-2340

11 del Pinal F. Technical tips for (dry) arthroscopic reduction and internal fixation of distal radius fractures. J Hand Surg [Am] 2011; 36: 1694-1705

12 Erhart S, Schmoelz W, Arora $R$ et al. The biomechanical effects of a deepend articular cavity during dynamic motion of the wrist joint. Clin Biomech (Bristol, Avon) 2012; 27: 557-561

13 Lutz M, Rudisch A, Kralinger $F$ et al. Sagittal wrist motion of carpal bones following intraarticular fractures of the distal radius. J Hand Surg [Br] 2005; 30: 282-287
14 Bednar JM. Arthroscopic treatment of triangular fibrocartilage tears. Hand Clin 1999; 15: 479-488

15 Geissler WB. Arthroscopically assisted reduction of intra-articular fractures of the distal radius. Hand Clin 1995; 11: 19-29

16 del Pinal F. Dry arthroscopy of the wrist: its role in the management of articular distal radius fractures. Scand J Surg 2008; 97: 298-304

17 Joseph SJ, Harvey JN. The dorsal horizon view: detecting screw protrusion at the distal radius. J Hand Surg [Am] 2011; 36: 1691-1693

18 Catalano LW $3^{\text {rd }}$, Cole RJ, Gelberman RH et al. Displaced intra-articular fractures of the distal aspect of the radius. Long-term results in young adults after open reduction and internal fixation. J Bone Joint Surg [Am] 1997; 79: 1290-1302

19 Catalano LW $3^{\text {rd }}$, Barron OA, Gickel SZ. Assessment of articular displacement of distal radius fractures. Clin Orthop Relat Res 2004; 423 : 79-84

20 Ono H, Furuta K, Fujitani R et al. Distal radius fracture arthroscopic intraarticular displacement measurement after open reduction and internal fixation from a volar approach. J Orthop Sci 2010; 15: 502-508

21 Auge WK $2^{\text {nd }}$, Velazquez PA. The application of indirect reduction techniques in the distal radius: the role of adjuvant arthroscopy. Arthroscopy $2000 ; 16$ : 830-835

22 Sammer DM, Shah HM, Shauver MJ et al. The effect of ulnar styloid fractures on patient-rated outcomes after volar locking plating of distal radius fractures. J Hand Surg [Am] 2009; 34: 1595-1602

23 Krämer S, Meyer H, O'Loughlin PF et al. The incidence of ulnocarpal complaints after distal radial fracture in the relation to the fracture of the ulnar styloid. J Hand Surg [Br] 2013; 38: 710-717

24 Giannoudis PV, Tzioupis C, Papathanassopoulos A et al. Articular stepoff and risk of post-traumatic osteoarthritis. Evidence today. Injury 2010; 41: 986-995 